\title{
Fluorescence Lifetime Distributions of Labeled Amorphous Polymers in Bulk
}

\author{
Berna Serrano, Juan BASElga, and Inés F. Piérola ${ }^{*, \dagger}$ \\ Instituto Tecnológico de Química y Materiales, Universidad Carlos III, 28911 Leganés, Spain \\ ${ }^{*}$ Dpto. de CC y TT Fisicoquimicas, Universidad a Distancia (UNED), C/ Senda del Rey 9, 28040 Madrid, Spain
}

(Received August 5, 2002; Accepted September 27, 2002)

\begin{abstract}
Polystyrene, poly(methylmethacrylate), poly(cyclohexyl methacrylate) and poly(1-butylmethacrylate) were labeled with anthryl groups by copolymerization. Films of labeled polymers as well as blends of them with unlabeled polystyrene samples of different polydispersity and molecular weight, were prepared by solvent casting. The time resolved emission of anthryl groups in those films was measured by Single Photon Counting with front face excitation at the standard $30^{\circ}$ incident angle and with much lower incident angles to photoselect chromophores on the polymer-air surface. Fluorescence decays were fitted with bimodal fluorescence lifetime distributions, which were analyzed taking into consideration some polymer characteristics and the compatibility of polymer blends. It was thus concluded that solid-like and liquid-like environments are both in the bulk and in the surface of the film although liquid-like domains are more frequent on the surface than in bulk. Polymer $T_{\mathrm{g}}$ determines the position of the two modes and polydispersity justify the broadness of the modes in the fluorescence lifetime distribution.

KEY WORDS Fluorescence Lifetime Distribution / Fluorescence Decay / Poly(1-butylmethacrylate) / Poly(methylmethacrylate) / Poly(cyclohexylmethacrylate) / Polystyrene /
\end{abstract}

Steady state or time-resolved fluorescence measurements of chromophores added to polymers as probes or labels can yield valuable information on the structural or dynamic properties of the system. ${ }^{1}$ Anthryl groups have been broadly employed as polymer labels due to its well known photophysical behavior as well as to its relatively small size and high fluorescence quantum yield. ${ }^{2,3}$ Nevertheless, it was found ${ }^{4}$ that the chain mobility of polystyrene (PS) is slightly reduced by the steric hindrance of the anthryl group with phenyl rings and therefore, minimum proportions of labeling groups must be introduced in the polymer to preserve unmodified the macroscopic polymer characteristics. Fortunately, due to the very high sensitivity of this technique, only very low chromophore concentrations are needed, which does not disturb the system properties. But even such low concentrations still report on its environment and on processes occurring in the nanosecond time range.

It was shown ${ }^{2}$ for anthracene dissolved in PS that rigidity and dimension of the matrix free volume are related with the vibronic structure of the fluorescence spectrum. The non-radiative deactivation processes, which involve out of plane vibrational modes and intermolecular interactions, are inhibited in polymer matrices. $^{2}$ As a consequence of the probe-polymer matrix interaction the fluorescence intensity changes with temperature following and revealing polymer relaxation processes. ${ }^{5}$ Matrix effects on the radiationless deactivation of substituted anthracenes were also observed in films of poly(methylmethacrylate) (PMMA) and they were attributed to competing intersystem crossing and very fast internal conversion. ${ }^{6}$ For 9 -methylanthracene, 7 the radiationless deactivation rate coefficients are dominated by Franck Condon factors in two types of coordinates: those of small groups such as protons with solvent independent dynamics and those of larger groups whose rate constants are inversely proportional to the viscosity showing relevant matrix effects.

Here we apply time-resolved fluorescence of anthryl labels to study the microscopic properties of PS and polymethacrylic polymers in the bulk amorphous state. The microstructure of a film made with a single amorphous polymer is expected to be homogeneous, above a certain length scale. Nevertheless, a single polymer is a polydisperse mixture formed by chains of different length that, in principle, i) may be interpenetrated totally or only to a certain extent, ${ }^{8-13}$ ii) may have preferential positions with respect to polymerair and support-polymer interfaces, ${ }^{14-16}$ iii) may have different proportions of liquid-like and solid-like domains, ${ }^{17-19}$ and that, even, iii) may show partially ordered domains. ${ }^{20-22}$ Thus, a certain heterogeneous microstructure must be expected even in the simplest polymer system: a single polymer sample in the bulk amorphous state. Several models have been proposed for the amorphous polymer state ${ }^{8-23}$ but currently, the most widely accepted considers that it is formed by interpenetrating random coils having unperturbed dimensions, as in $\theta$ solvents. ${ }^{23}$ 
Only a few techniques give information on the microstructure of bulk amorphous polymer samples, at the molecular level. Among them, light, ${ }^{18}$ electron, ${ }^{22}$ neutron, ${ }^{24,25}$ and X-Ray ${ }^{24}$ scattering, electron spin resonance ${ }^{19}$ or diffraction and neutron reflectometry ${ }^{16,26}$ are the most widely used. Time resolved fluorescence measurements, may also provide useful information on the microstructure of polymer samples in bulk. ${ }^{13,16,17,27}$

Common fluorescent molecules in non-viscous homogeneous dilute solution and in the absence of excimer or exciplex formation give single exponential decays in the nanosecond time range. Such is the case of 9-anthrylmethylmethacrylate (MMA-An) in dioxane dilute solution which yields a fluorescence lifetime of $4.56 \mathrm{~ns},{ }^{13}$ a very close value to that previously reported for the model compound, 9-methylanthracene, $4.6 \mathrm{~ns}^{28}$ Fitting of the MMA-An emission in dilute solution to a fluorescence lifetime distribution worsens the statistical fitting parameters and the trend of the residuals with respect to fittings with discrete number of exponentials. Meanwhile, as shown below, the opposite occurs with labeled polymer samples in bulk, fitting with fluorescence lifetime distributions yields better statistical parameters than mono or biexponential fittings. This experimental fact may be interpreted as due to the different environments experienced by the excited chromophore in both situations. In solution, only an average environment (solvent) is experienced by the chromophore, probably reflecting that the solvent cage re-equilibration process after excitation occurs in a time range much smaller than the chromophore fluorescence lifetime $(\tau)$. In a solid polymer matrix below $T_{\mathrm{g}}$, the label may be placed in a set of not too different environments but since dynamics are severely restricted, no thermal averaging occurs; the distribution of fluorescence lifetimes probably reflects a distribution of sites. Therefore, the analysis of the fluorescence lifetime distribution of a chromophore may be a suitable tool to obtain information about some characteristics of the environments surrounding a segment of a polymer chain.

In many heterogeneous systems at the nanoscale, continuous distributions of fluorescence lifetimes are the most likely representation of the multiplicity of interactions taking place in different environments. ${ }^{29}$ Probes in micelle systems or cyclodextrin cavities, chromophores adsorbed on surfaces, tryptophan residues in biological systems and Förster electronic energy transfer in rigid media are some examples of systems being analyzed with distributions of fluorescence lifetimes, provided in ref 29. Many other examples have been published later. ${ }^{30-32}$ Labeled polymers in bulk are obviously good candidates to this type
Table I. Characteristics of the polymer samples: weight average molecular weight $\left(M_{\mathrm{w}}\right)$ and polydispersity $(r)$ determined by SEC in THF, intrinsic viscosity $([\eta])$ measured in THF at $30^{\circ} \mathrm{C}$, and mole fraction of labeling monomer in the copolymers $\left(F_{\mathrm{An}}\right)$

\begin{tabular}{lcccc}
\hline & $M_{\mathrm{w}} \times 10^{-3}$ & $r$ & {$[\eta]\left(\mathrm{dL} \mathrm{g}^{-1}\right)$} & $F_{\text {An }}(\mathrm{mol} \%)$ \\
\hline PS4 & 4.0 & 1.07 & - & 0 \\
PS19 & 19.3 & 1.07 & - & 0 \\
PS44 & 44.1 & 1.07 & - & 0 \\
PS24 & 24.0 & 1.85 & 0.175 & 0 \\
PS-An & 23.6 & 1.89 & 0.166 & 0.099 \\
PMMA-An & 22.7 & 1.67 & 0.143 & 0.103 \\
PBuMA-An & 22.3 & 1.57 & 0.102 & 0.135 \\
PChMA-An & 171 & 4.7 & 0.175 & 0.204 \\
\hline
\end{tabular}

of analysis since distributions carry a physical meaning easier to be understood than discrete exponentials for such microheterogeneous systems. In the following, we will show the influence on the fluorescence lifetime distribution (FLD) of labeled amorphous polymers, of important polymer characteristics such as polydispersity, glass transition temperature or situation in the surface or in bulk. The objective was to determine the physical properties of the film that can be deduced from the analysis of FLD.

\section{EXPERIMENTAL}

Polystyrene (PS-An), poly(methylmethacrylate) (PMMA-An), poly(n-butyl methacrylate) (PBuMAAn) and poly(cyclohexyl methacrylate) (PChMA-An) labeled with anthryl groups (An) by copolymerization of the corresponding monomer with $0.3-0.4 \mathrm{wt} \%$ (9-anthrylmethyl)methacrylate (Polysciences), were synthesized by radical polymerization in dioxane solution, at $80^{\circ} \mathrm{C}$, with AIBN as initiator. The total monomer concentration in the feeding was $30.3 \% \mathrm{w} / \mathrm{v}$ for PS-An and $15 \% \mathrm{w} / \mathrm{v}$ for polymethacrylics. Labeled polymers were purified by repeated precipitations until the fluorescence emitted by free monomer was less than $0.1 \%$ of the emission from the labeled polymer, as observed by SEC with fluorescence detection. Unlabeled polydisperse polystyrene (PS24) was synthesized in the same conditions as PS-An. Three more samples of unlabeled PS were employed: PS4, PS19, and PS44; they were monodisperse standards purchased from Aldrich and used as received.

Table I summarizes the characteristics of the samples employed in this work. The chromophore mole fraction in the copolymers $\left(F_{\mathrm{An}}\right)$ was determined by spectrophotometry (Perkin-Elmer Lambda 6) in dioxane solution. Polymer molecular weights were determined by SEC (Hewlett Packard-Waters) in THF at $30^{\circ} \mathrm{C}$, using polystyrene standards (Polysciences). Intrinsic viscosities in THF at $30^{\circ} \mathrm{C}$, were measured in an 
automatic capillary viscometer (Lauda Viscometer).

Solutions of single labeled polymers or blends of $50 \mathrm{wt} \%$ PS and one of the labeled polymers were prepared in THF with $11 \mathrm{wt} \%$ total polymer concentration. Solutions were cast at $50{ }^{\circ} \mathrm{C}$ on low fluorescence microscope slides previously silanized with methyltrichlorosilane. The resulting films were circular, with diameters of about $8 \mathrm{~mm}$ and $600 \mu \mathrm{m}$ thick. The border of the film, a crown of about $2 \mathrm{~mm}$, was excluded from observations. It was verified that films prepared in this way give place to the same fluorescence results than annealed and slowly cooled samples.

Fluorescence decay measurements were performed with a time-correlated single photon counting technique from Edinburgh Instruments ( $\mathrm{nF} 900)$, provided with a nitrogen lamp pulsed at $40 \mathrm{kHz}$ and operating at $6.3 \mathrm{kV}$. The excitation wavelength was set at $355 \mathrm{~nm}$ and the emission one at $420 \mathrm{~nm}$. The glass plates with the polymer blend adhered on the surface were placed in the sample holder forming an angle with the incident beam equal to $30^{\circ}$, except when indicated. Slits and focal length of excitation beam were adjusted to detect $1 \%$ of lamp counts. Lamp profile acquisitions were intercalated each 2000 counts of the sample decay and were obtained with light reflected by the sample glass support. Counts collection was stopped when the number of counts in the maximum was $2 \times 10^{4}$. Standard deconvolution routines (Edinburgh Instruments, Level 2) were used to obtain multiexponential fitting functions presented as fluorescence lifetime distributions (FLD).

\section{RESULTS AND DISCUSSION}

Bulk samples containing polymers labeled with An groups were prepared and their fluorescent emission was measured with front face excitation. No excimer emission of An was observed in the steady state fluorescence spectra of any of the studied samples and therefore, it must be concluded that no aggregation of chromophores takes place and consequently, the labeled chains are well dispersed within the bulk sample. The low degree of labeling (Table I) ensures that the structure of the polymer system is not modified with respect to the unlabeled system prepared in the same experimental conditions.

Figure 1 presents a representative decay of the fluorescence emitted by An groups anchored to PS or to the polymethacrylic polymers here studied. The best fit was attained in all cases with FLD, while fitting with a discrete number of exponential functions worsens the statistical parameters $\left(\chi^{2}\right)$ and the trend of the residuals.
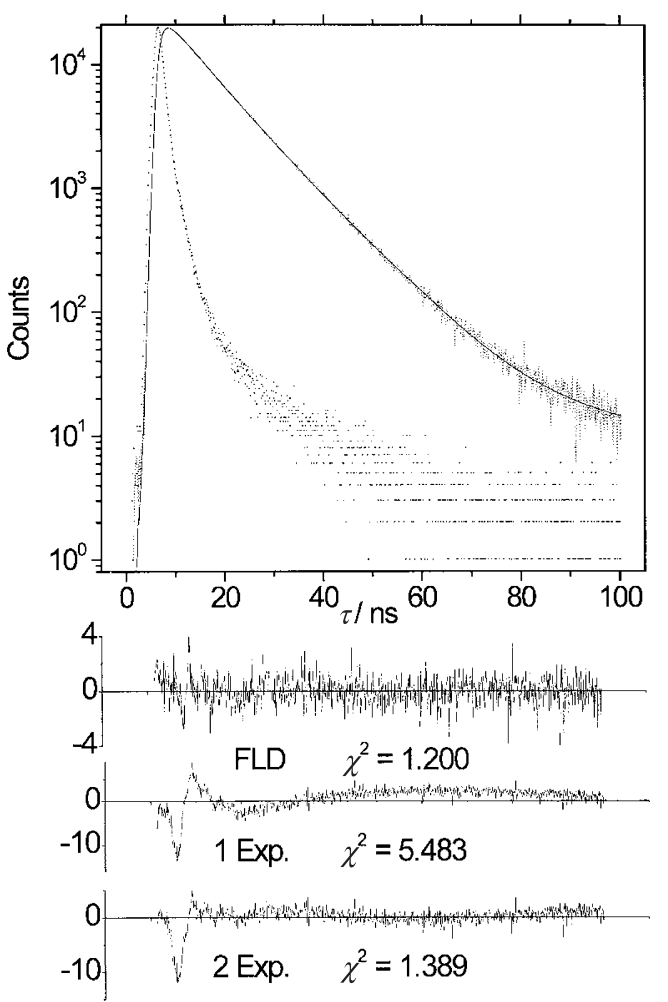

Figure 1. Typical lamp profile and fluorescence decay of An anchored to PS (dots) and best fit (line). Residuals corresponding to fits with discrete number of exponentials and FLD are also plotted below.

FLD were bimodal in most cases here studied. That is a common result $\mathrm{t}^{29-32}$ not only for polymer films but also for many other different systems mentioned above, which suggests that bimodality is something intrinsic to the analytical method or something in common to the different systems, for example, the different properties of bulk and surface. Bimodality, shape and shift of FLD to lower or larger fluorescence lifetimes (always below $13.5 \mathrm{~ns}$, the natural fluorescence lifetime of An), ${ }^{28}$ are likely dependent on the different characteristics of the multiplicity of An environments that may affect the radiationless electronic transition probability of An in the film. As pointed out above, several of those different physical properties which may differentiate two types of environments could be: interfacial or bulk positions with different access to quenchers, locally strained positions, solid-like and liquid-like domains coexisting below $T_{\mathrm{g}},{ }^{17}$ or regions with different free volume due, for example, to different proportions of chain-ending groups. Among those physical properties of the environment only some of them may control the photophysics of An groups: local viscosity or rigidity. ${ }^{5-7}$ It is known that in amorphous polymers some flexible-like or liquid-like sites where side chain motions are allowed, may coexist, far below the $T_{\mathrm{g}}$, with solid-like domains. From the analysis of the 
Table II. Characteristics of FLD of An labeled polymer of different $T_{\mathrm{g}}:{ }^{36}$ average fluorescence lifetime ( $<\tau>$ in $\left.\mathrm{ns}\right)$, full width at half maximum ( $f w h m$, in ns), relative contribution of low $\tau$ mode $A_{1}$ and statistical fitting parameter $\chi^{2}$

\begin{tabular}{lccccccc}
\hline SAMPLE & $\chi^{2}$ & $\left\langle\tau>_{1}\right.$ & $f w h m_{1}$ & $A_{1}(\%)$ & $\langle\tau\rangle_{2}$ & $f w h m_{2}$ & $T_{\mathrm{g}} /{ }^{\circ} \mathrm{C}$ \\
\hline PMMA-An & 1.200 & 5.20 & 1.13 & 14.8 & 9.34 & 4.49 & 107 \\
PS-An & 1.199 & 4.54 & 1.12 & 13.6 & 8.46 & 4.32 & 100 \\
PChMA-An & 1.138 & 4.67 & 1.78 & 39.0 & 8.08 & 2.96 & 73 \\
PBuMA-An & 1.198 & 2.74 & 1.12 & 18.9 & 6.48 & 4.35 & 30 \\
\hline
\end{tabular}

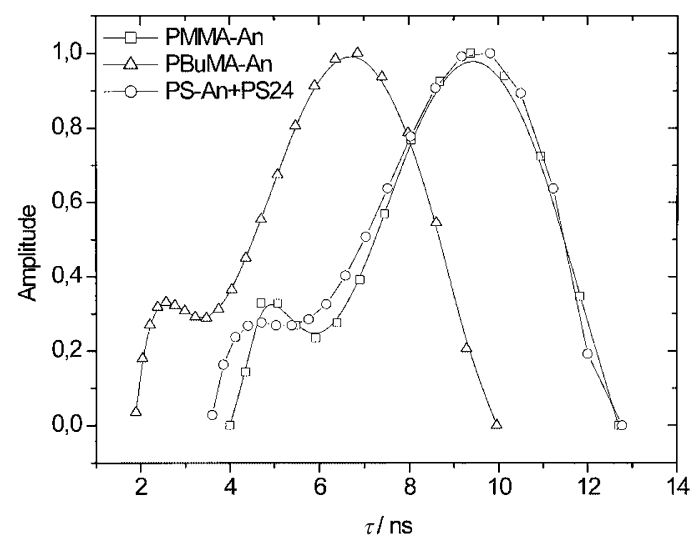

Figure 2. Distributions of An fluorescence lifetime in polymer samples with different $T_{\mathrm{g}}$ : PMMA-An $\left(T_{\mathrm{g}}=107^{\circ} \mathrm{C}\right)$, PS-An $\left(T_{\mathrm{g}}=\right.$ $\left.100^{\circ} \mathrm{C}\right)$ and PBuMA-An $\left(T_{\mathrm{g}}=30^{\circ} \mathrm{C}\right) .{ }^{36}$

fluorescence decay of a probe (malachite green) in several polymer matrices, with biexponential functions, it was concluded ${ }^{17}$ that the fast decay component arises from sites where phenyl or ester side groups freely rotate in the matrix with relatively small activation energy and therefore small changes with decreasing temperature with respect to $T_{\mathrm{g}}$. The component with larger $\tau$ was assigned to solid-like domains of larger rigidity. A similar interpretation may be applied to the two modes here observed in FLD of An labels, in such a way that, the mode located in the low $\tau$ side may be related with the response of An located in liquid-like domains and the high $\tau$ mode, predominant in the FLD, may correspond to solid-like sites.

The dependence of FLD on $T_{\mathrm{g}}$ supports that assignment. $T_{\mathrm{g}}$ (Table II) seems to be quite determinant of the position of the two modes if we compare the FLD of three polymer samples having practically the same molecular weight distribution (MWD) and very different $T_{\mathrm{g}}$, as shown in Figure 2. Increasing $T_{\mathrm{g}}$ (from PBuMA-An to PMMA-An) the whole FLD shifts to larger $\tau$. Table II summarizes the relevant characteristics of the FLD modes: position of the maxima or average fluorescence lifetime $\langle\tau\rangle_{i}$, full width at half maximum $f w h m_{\mathrm{i}}$ and relative proportion in the overall integral $A_{i}$. It is remarkable that fwhm of the two modes is practically identical for the three polymers having the same MWD whereas it is different for PChMA-An

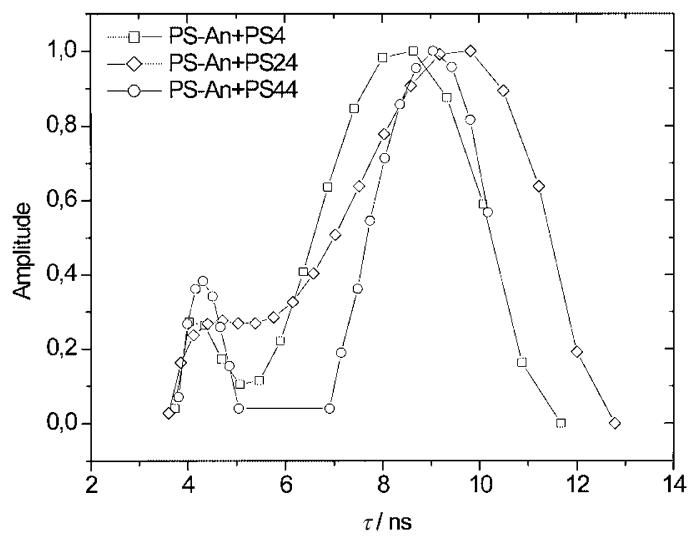

Figure 3. Distributions of An fluorescence lifetime in samples with $50 \mathrm{wt} \%$ PS-An and unlabeled PSx of different molecular weights (Table I).

with a totally different MWD (see Table I). From Table II results it may be concluded that i) $\langle\tau\rangle$ depends on the sample $T_{\mathrm{g}}$ whereas ii) fwhm is independent on the polymer $T_{\mathrm{g}}$ but strongly dependent on MWD and iii) the relative contribution of the two modes $A_{i}$ depends on both $T_{\mathrm{g}}$ and MWD.

Bimodal MWD of PS were obtained by mixing $50 \mathrm{wt} \%$ PS-An with monodisperse PS having a molecular weight lower (PS-An+PS4) or larger (PSAn+PS44) than labeled PS. Figure 3 compares the FLD of those bimodal mixtures and a monomodal MWD mixture, PS-An+PS24, obtained with a PS sample of about the same MWD than labeled PS. The positions of the mode assigned to liquid-like domains are not very different in the three FLD but its relative contribution is slightly larger for the sample PS-An+PS44 indicating a segregation of labeled PS to liquid-like domains induced by the presence of a larger proportion of high molecular weight components (PS44) in the MWD. Some differences in the fwhm can also be observed in Figure 3, in particular in the high $\tau$ or solidlike mode. It seems so, that polydispersity plays an important role in determining the width and proportion of modes in FLD, low molecular weight chains with larger mobility, because of larger proportion of ending groups, should have a larger contribution to liquid-like domains and besides, they give place to broader distribution of $T_{\mathrm{g}}$ and broader FLD.

Low molecular weight chains of PS have a preferential position in the film surface with respect to the bulk $^{33}$ and it is more pronounced in bimodal MWD with larger disparity between the two components. Trying to distinguish between fluorescence coming from the surface and from bulk, we have prepared incompatible blends of PS with PMMA or PBuMA which are opaque and thus, fluorescence coming mainly from surface may be observed. In such incompatible blends the 


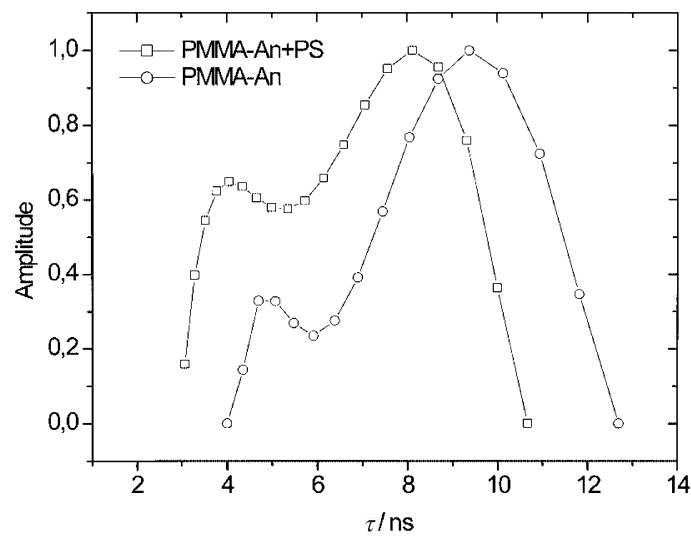

Figure 4. Distributions of An fluorescence lifetime in PMMAAn and in opaque incompatible blends of $50 \mathrm{wt} \%$ PS24 with PMMA-An.

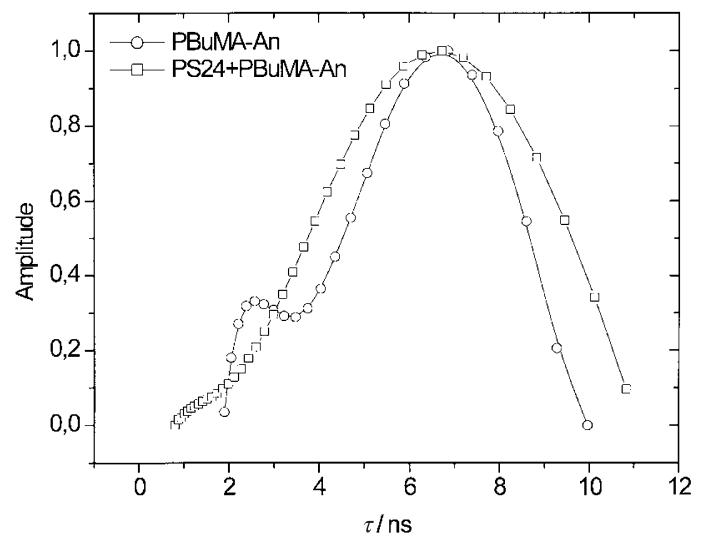

Figure 5. Distributions of An fluorescence lifetime in PBuMA-An and in opaque incompatible blends of $50 \mathrm{wt} \%$ PS24 with PBuMA-An.

label is confined in homopolymer domains and therefore the environment of the label will be the same as in the single polymer sample, except for the position in the film (interfaces, bulk or surface) or the presence of stresses induced by surface tension. Figure 4 and 5 compare the FLD of An in the single labeled polymer (PMMA-An or PBuMA-An) and in the incompatible blends with $50 \mathrm{wt} \%$ PS. It may be observed that in the surface of the opaque blend PMMA-An+PS, i) the FLD is shifted to lower $\tau$ with respect to pure PMMAAn, as corresponding to lower $T_{\mathrm{g}}$ in the surface than in bulk, the last one being observed in the thick PMMAAn sample where the surface contribution must be a minor contribution, ii) the proportion of liquid-like domains in the surface increases with respect to the bulk and iii) fwhm practically does not change, probably because the MWD of the labeled polymer is about the same in the phase-separated surface and in bulk. These results may be easily understood since it is well known $^{33}$ that $T_{\mathrm{g}}$ in the surface of a polymer film is lower than in the bulk and therefore a larger proportion of liquid-like domains must be expected in the sur-

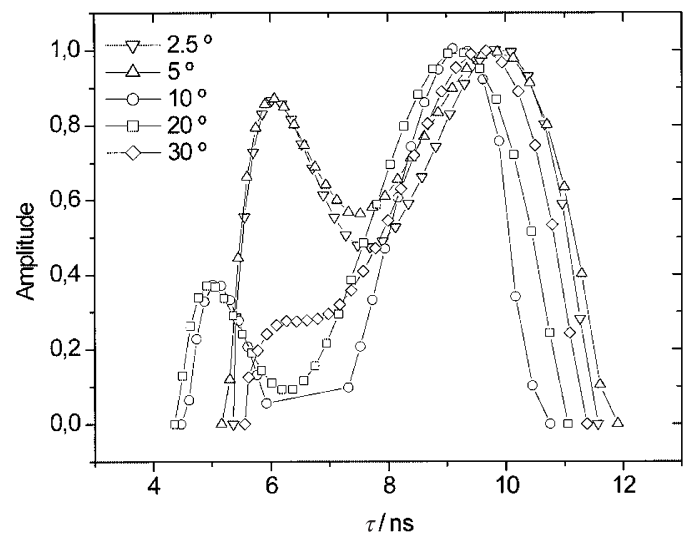

Figure 6. Distributions of An fluorescence lifetime in sample $50 \mathrm{wt} \%$ PS19+PS-An obtained with different incident angles.

face but Figure 5 shows a slightly different behavior for PBuMA-An and PBuMA-An+PS: the liquid-like mode is shifted in the blend to lower $\tau$, as before, but the solid-like mode enlarges and the expected increment of the contribution of liquid-like sites is not observed. This points that polymer-polymer interfaces play an important role in the surface of the phase separated PBuMA-An+PS blend in such a way that liquidlike domains of PBuMA-An diminish and the range distribution of PBuMA-An solid-like domains enlarges through the presence of the more rigid PS.

An other attempt to distinguish between the surface and bulk fluorescence was made by photoselecting the surface using extremely low incident angles of the excitation beam on the film. Figure 6 plots the obtained FLD. The most striking result was that with the lowest incident angles the contribution of the small $\tau$ or liquidlike mode increases markedly, although it does not become the only contribution. The presence of the two modes may be associated with the interference of fluorescence coming from bulk or with both solid-like and liquid-like domains being present in the surface. Surprisingly, the position of the two modes shifts to larger $\tau$, as if the surface would have larger $T_{\mathrm{g}}$ (rigidity) than the bulk. The opposite (smaller $T_{\mathrm{g}}$ ) has been reported to occur at the surface ${ }^{33}$ of PS samples with Mn lower than $30 \mathrm{k}$, what was assigned to chain ends segregation. In addition, lower $\tau$ in the surface than in bulk should also be expected taking into account that labels at the polymer-air surface may be quenched by oxygen more easily than in bulk. By the other hand, the same result than here reported, larger $\tau$ in the surface than in bulk, was observed for pyrene probes deposited on a polymer surface by sublimation of the chromophore ${ }^{34}$ or in LB films in which pyrene probes motion was highly restricted so that $\tau$ were longer than those in THF solution. ${ }^{35}$ This leaves an open question that will be studied in future work. 


\section{CONCLUSIONS}

Fluorescence decay of An labels anchored to PS, PMMA, PChMA, and PBuMA may be fitted with bimodal FLD whose characteristics depend on several properties of the polymer sample: the position on the two modes depends directly on the sample $T_{\mathrm{g}}$, their fwhm is controlled by polydispersity and the integral contribution of the modes to the total FLD depends on both $T_{\mathrm{g}}$ and MWD. For transparent samples and using standard incident angles, the fluorescence observed comes mostly from the labeled chains in bulk and the mode corresponding to larger $\tau$ is dominant. When using grazing incident angles it may be assumed that the observed fluorescence comes mostly from the surface of the film and then, the contribution of the mode corresponding to lower $\tau$ increases significantly. These results suggest that the lower $\tau$ mode corresponds to liquid-like domains, more frequent in the surface than in bulk, whereas the larger $\tau$ mode would correspond to more rigid solid-like domains. Opaque incompatible blends do also show fluorescence from the surface but the presence of polymer-polymer interfaces may modify the proportion of liquid-like domains with respect to the surface of the single labeled polymer.

Acknowledgments. Financial support from DGI (Spain) under grants BQU2000-0251 and MAT20000391-P4-02, is gratefully acknowledged.

\section{REFERENCES}

1. J. R. Lakowicz, "Principles of Fluorescence Spectroscopy", 2nd ed, Kluwer Academic/Plenum Publishing, New York, N.Y., 1999, chapt. 14.

2. L. Coltro, D. Dibbern-Brunelli, C. A. B. Elias, M. Talhavini, M. G. de Oliveira, and T. D. Z. Atvars, J. Braz. Chem. Soc., 6, 127 (1995).

3. F. Wilkinson, D. R. Worrall, and S. L. Williams, J. Phys. Chem., 99, 6689 (1995).

4. J. Horinaka, S. Ito, M. Yamamoto, Y. Suhii, and T. Matsuda, Macromolecules, 32, 2270 (1999).

5. T. D. Z. Atvars, A. P. Dorado, and I. F. Pierola, Polym. Netw. Blends, 7, 111 (1997).

6. S. Schoof and H. Guesten, Ber. Bunsen-Ges. Phys. Chem., 93, 864 (1989).

7. E. Blatt, F. E. Treloar, K. P. Ghiggino, and R. G. Gilbert, J. Phys. Chem., 85, 2810 (1981).

8. M. Vacatello, D. Y. Yoon, and P. J. Flory, Macromolecules, 23, 1993 (1990).

9. D. J. Worsfold, J. Polym. Sci., Polym. Phys. Ed., 15, 103 (1977).

10. P. H. Lindenmeyer, J. Macromol. Sci., Phys., 8, 361 (1973).
11. B. Vollmert, "Polymer Chemistry", Springer-Verlag, Berlin, 1973, p 552.

12. G. V. Gozlov and V. U. Novikov, Phys. Uspekhi, 44, 681 (2001).

13. B. Serrano, J. Baselga, M. R. Vigil, and I. F. Pierola, submitted.

14. Y. Pu, S. Ge, M. Rafailovich, J. Sojolov, Y. Duan, E. Pearce, V. Zaitsev, and S. Schwarz, Langmuir, 17, 5865 (2001).

15. T. Kajiyama, N. Satomi, K. Tanaka, and A. Takahara, Macromol. Symp., 143, 171 (1999).

16. A. Hariharan and S. K. Kumar, J. Chem. Phys., 98, 4163 (1993).

17. J. Y. Ye, T. Hattori, and H. Nakatsuka, Phys. Rev. B: Condens. Matter Mater. Phys, 56, 5286 (1997).

18. C. T. Moynihan and J. Schroeder, J. Non-Cryst. Solids, 160, 52 (1993).

19. J. I. Spielberg and E. Gelerinter, Phys. Rev. B, 30, 2319 (1984).

20. R. Zhang and W. L. Mattice, Macromolecules, 28, 7454 (1995).

21. V. P. Privalko and Y. S. Lipatov, Makromol. Chem., 175, 641 (1974).

22. K. C. Honnell, J. D. McCoy, J. G. Curro, K. C. Schweizer, A. H. Narten, and A. Habenshuss, J. Chem. Phys., 94, 4659 (1991).

23. P. J. Flory, Faraday Discuss. Chem. Soc., 68, 14 (1979).

24. J. P. Cotton, D. Decker, H. Benoit, B. Farnoux, J. Higgins, G. Jannink, R. Ober, C. Picot, and J. desCloizeaux, Macromolecules, 7, 863 (1974).

25. G. D. Wignall, D. G. Ballard, J. Schelten, Eur. Polym. J., 10, 861 (1974).

26. C. J. Clarke, R. A. L. Jones, J. Edwards, and J. Penfold, Macromolecules, 28, 2048 (1995).

27. T. D. Z. Atvars, I. Esteban, B. Illera, B. Serrano, M. R. Vigil, and I. F. Pierola, J. Lumin., 72-74, 467 (1997).

28. I. Berlman, "Handbook of Fluorescence Spectroscopy of Aromatic Molecules", 2nd ed, Academic Press, Inc. New York, N.Y., 1971, p 358.

29. W. R. Ware, "Photochemistry in Organized and Constrained Media", R. Ramamurthy, Ed., VCH Publishers, New York, N.Y, 1991, chapt. 13.

30. J. Y. Ye, M. Ishikawa, O. Yogi, T. Okada, and Y. Maruyama, Chem. Phys. Lett., 288, 885 (1998).

31. J. A. Lacey and D. Phillips, Photochem. Photobiol. Sci., 1, 378 (2002).

32. J. Feng, M. A. Winnik, and A. Siemiarczuk, J. Polym. Sci., Part B: Polym., Phys., 36, 1115 (1998).

33. a) K. Tanaka, T. Kajiyama, A. Takahara, and S. Tasaki, Macromolecules, 35, 4702 (2002).

b) T. Kajiyama, K. Tanaka, and A. Takahara, Polymer, 39, 4665 (1998).

34. E. A. Prado, S. B. Yamaki, T. D. Z. Atvars, O. E. Zimerman, and R. G. Weiss, J. Phys. Chem. B, 104, 5905 (2000).

35. J. Matsui, M. Mitsuishi, and T. Miyashita, J. Phys. Chem. B, 106, 2468 (2002).

36. J. Brandrup, E. H. Immergut, and E. A. Grulke, D. Bloch, and A. Abe, "Polymer Handbook", 4th ed., John Wiley \& Sons. Inc., New York, N.Y., 1999. 